\title{
Expression, purification and characterization of soluble red rooster laforin as a fusion protein in Escherichia coli
}

\author{
M Kathryn Brewer, Satrio Husodo, Vikas V Dukhande, Mary Beth Johnson and Matthew S Gentry*
}

\begin{abstract}
Background: The gene that encodes laforin, a dual-specificity phosphatase with a carbohydrate-binding module, is mutated in Lafora disease (LD). LD is an autosomal recessive, fatal progressive myoclonus epilepsy characterized by the intracellular buildup of insoluble, hyperphosphorylated glycogen-like particles, called Lafora bodies. Laforin dephosphorylates glycogen and other glucans in vitro, but the structural basis of its activity remains unknown. Recombinant human laforin when expressed in and purified from $E$. coli is largely insoluble and prone to aggregation and precipitation. Identification of a laforin ortholog that is more soluble and stable in vitro would circumvent this issue.
\end{abstract}

Results: In this study, we cloned multiple laforin orthologs, established a purification scheme for each, and tested their solubility and stability. Gallus gallus (Gg) laforin is more stable in vitro than human laforin, Gg-laforin is largely monomeric, and it possesses carbohydrate binding and phosphatase activity similar to human laforin.

Conclusions: Gg-laforin is more soluble and stable than human laforin in vitro, and possesses similar activity as a glucan phosphatase. Therefore, it can be used to model human laforin in structure-function studies. We have established a protocol for purifying recombinant Gg-laforin in sufficient quantity for crystallographic and other biophysical analyses, in order to better understand the function of laforin and define the molecular mechanisms of Lafora disease.

Keywords: Laforin, Lafora disease, Phosphatase, Carbohydrate-binding module, Glycogen

\section{Background}

Lafora disease (LD) is an autosomal recessive, neurodegenerative disorder resulting in myoclonus, epilepsy, dementia, and death [1-3]. Affected individuals experience an initial seizure during adolescence, followed by severe neurological decline until the patient's death approximately ten years after the first seizure $[1,4]$. Characteristic of the disease is the cytoplasmic accumulation of hyperphosphorylated glycogen-like particles called Lafora bodies (LBs) in various tissues including brain, muscle and liver [1,5].

Approximately $50 \%$ of Lafora disease cases are caused by mutations in the EPM2A (epilepsy of progressive myoclonus type 2 gene A) gene that encodes the protein laforin [4-6]. EPM2A is conserved in all vertebrate genomes, but it is absent from the genome of most non-

\footnotetext{
* Correspondence: matthew.gentry@uky.edu

Department of Molecular and Cellular Biochemistry and Center for Structural Biology, College of Medicine, University of Kentucky, 741 S. Limestone, Lexington, Kentucky 40536-0509, USA
}

vertebrate organisms including standard model organisms such as Saccharomyces cerevisiae, Caenorhabditis elegans, and Drosophila melanogaster [7,8]. An exception to this rule is a small subgroup of protists that synthesize floridean starch, an insoluble carbohydrate similar to LBs. Five protozoan laforin orthologs have been identified; however, sequence identity between these proteins and human laforin is $<37 \%$ and the genes have major insertions and deletions $[7,8]$. Thus, these proteins are not optimal orthologs to utilize for modeling human laforin.

Laforin is a bimodular protein with a carbohydratebinding module (CBM) at its amino-terminus and a dual-specificity phosphatase (DSP) domain at its carboxyterminus [9-11]. CBMs are most commonly found in glycosyl hydrolases and glucosyl transferases from bacteria, fungi or plants, and there are over 39 families of CBMs that bind a variety of carbohydrate substrates. Laforin belongs to the CBM20 family according to the Carbohydrate-Active Enzymes (CAZy; www.cazy.org) database [12]. CBM20s are 
closely related to CBM48s, and both are classified as starch-binding domains with similar folds and binding sites [13-15]. Typical of DSPs, laforin is capable of hydrolyzing phosphotyrosine and phosphoserine/phosphothreonine substrates; however, laforin is unique among phosphatases in that it is the only phosphatase in humans containing a CBM, which targets laforin to glycogen [8,9]. Laforin has been shown to bind and dephosphorylate glycogen and other glucans in vitro and in vivo [8,9,16-19].

Glycogen is an energy storage molecule synthesized by bacterial, fungal and animal species consisting of $\alpha-1,4$ and $\alpha-1,6$ linked residues of glucose, with $12-14$ residues per branch [20]. Glycogen has been shown to contain small amounts of phosphate, but the regulation and effects of this phosphorylation event are currently under debate [19,21-25]. While the source of phosphorylation is disputable, data from multiple labs has clearly established that loss of laforin activity results in hyperphosphorylation and poorly branched glycogen, resulting in insoluble LBs [17-19,23,26].

Although the substrate and function of laforin have recently been elucidated, the structural basis for the unique glucan phosphatase activity of laforin remains unknown. Ourselves and others have experienced difficulty purifying laforin in sufficient quantities and of sufficient quality for crystallographic studies [27]. One group recently demonstrated that recombinant human laforin expressed in $E$. coli is largely insoluble and must be purified from inclusion bodies [27]. This procedure requires denaturation and refolding steps, involves harsh chemical treatments, and often yields low amounts of correctly folded protein. A subsequent report demonstrated that only the laforin CBM was soluble when expressed in E. coli [28].

Our lab has purified enough recombinant laforin from the soluble portion of bacterial cell lysates to perform in vitro assays [8,16,29-31]. However, the protein often aggregates and precipitates after the multistep purification procedure. In this study, we found that the addition of sugars to the lysis and purification buffers increases the yield of soluble laforin from lysates and improves stability. However, such additives interfere with methods such as isothermal titration calorimetry that directly measure protein-ligand interactions. Also, we have been unable to crystallize laforin purified in the presence of sugars (unpublished data). Our group recently determined the structures of two glucan phosphatases from Arabidopsis that are functionally similar to laforin, and the structures of other DSP domains and CBMs are available [32,33]. However, these structures provide little information about the function of laforin due to low similarity between these domains and the domains of laforin. We then sought a laforin ortholog that is highly similar to human laforin (Hs-laforin) and, when expressed in bacteria, is less prone to aggregation and precipitation. We cloned and purified multiple laforin orthologs and optimized the purification of recombinant Gallus gallus laforin (Gg-laforin). Previously, the CBM of Gg-laforin was fused to a glutathione $S$-transferase (GST) tag and shown to bind glycogen [34]. In this study, we purified SUMO-tagged full-length Gg-laforin and confirmed that Gg-laforin functions as a monomer, contrary to prior claims that laforin dimerization is necessary for phosphatase activity $[27,35]$. Phosphatase and glucan binding assays indicate that the catalytic and binding ability of Gg-laforin is comparable to that of Hs-laforin [8,30,36]. Therefore, Gg-laforin is an excellent model for Hs-laforin and a better alternative for crystallization and other biophysical studies.

\section{Results and discussion}

\section{Instability of Hs-laforin and other laforin orthologs}

Soluble Hs-laforin has proved to be a difficult protein to purify from E. coli $[27,28]$. While we have successfully purified some Hs-laforin suitable for in vitro assays, the protein is unstable and precipitates from solution. Thus, we sought to optimize the purification procedure using an additive. His $_{6}$-tagged Hs-laforin was expressed and purified from $E$. coli by affinity chromatography. Approximately $5 \mathrm{mg}$ of soluble Hs-laforin was obtained from $1 \mathrm{~L}$ of $E$. coli cells. In order to increase the solubility of Hs-laforin, we tested the addition of the sugars maltose and $\beta$-cyclodextrin (BCD) to the purification buffer. The addition of $15 \%$ maltose (w/v) or $10 \mathrm{mM}$ $\mathrm{BCD}$ to the lysis and purification buffers improved the yield of soluble Hs-laforin to $8 \mathrm{mg}$ and $9 \mathrm{mg}$ per $1 \mathrm{~L}$ culture, respectively (Figure $1 \mathrm{~A})$. Next we sought to define the stability of recombinant Hs-laforin purified in the different buffers using two methods.

We first determined the stability of Hs-laforin by concentrating the protein using centrifugal filter units and measuring the volume and concentration throughout the centrifugation process. The Hs-laforin preparation without added sugars did not exceed $5 \mathrm{mg} / \mathrm{ml}$ and total soluble protein was reduced by $37 \%$ during the centrifugation process (Figure 1B). Conversely, Hs-laforin purified in the presence of maltose or BCD was concentrated to $>11 \mathrm{mg} /$ $\mathrm{ml}$, and total soluble protein content was reduced by less than $21 \%$ (Figure 1B). Thus, the addition of BCD or maltose allows Hs-laforin to be concentrated to higher concentrations likely by preventing aggregation and precipitation. Second, we sought to define the long-term stability of Hs-laforin +/- sugars. Hs-laforin was incubated at room temperature and protein concentrations were measured over a period of eight days. After only 12 hours, the concentration of Hs-laforin had fallen significantly and continued to drop over the eight-day period (Figure $1 \mathrm{C}$ ). With the addition of maltose, the concentration did not 


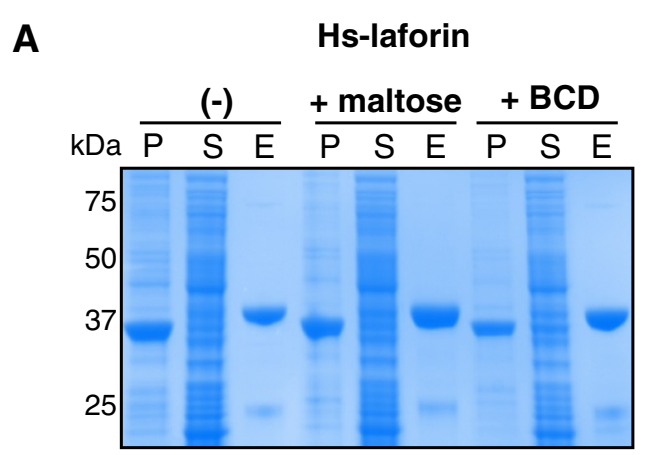

B

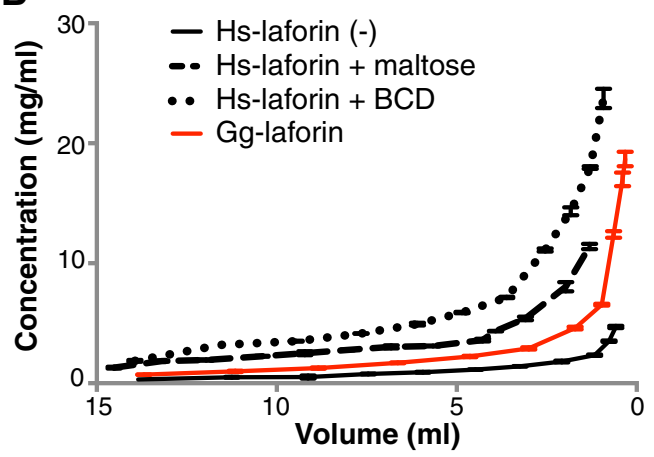

\begin{tabular}{|c|c|c|c|c|}
\hline $\begin{array}{l}\text { protein }+ \\
\text { additive }\end{array}$ & $\begin{array}{l}\text { initial total } \\
\text { protein } \\
\text { (mg) }\end{array}$ & $\begin{array}{l}\text { final total } \\
\text { protein } \\
\text { (mg) }\end{array}$ & $\%$ reduc & $\begin{array}{c}\text { final } \\
\text { d concentration } \\
(\mathrm{mg} / \mathrm{ml})\end{array}$ \\
\hline Hs-laforin (-) & 4.29 & 2.72 & $37 \%$ & 4.69 \\
\hline Hs-laforin + maltos & se 19.02 & 14.94 & $21 \%$ & 11.41 \\
\hline Hs-laforin + BCD & 26.11 & 21.84 & $16 \%$ & 23.74 \\
\hline
\end{tabular}

C

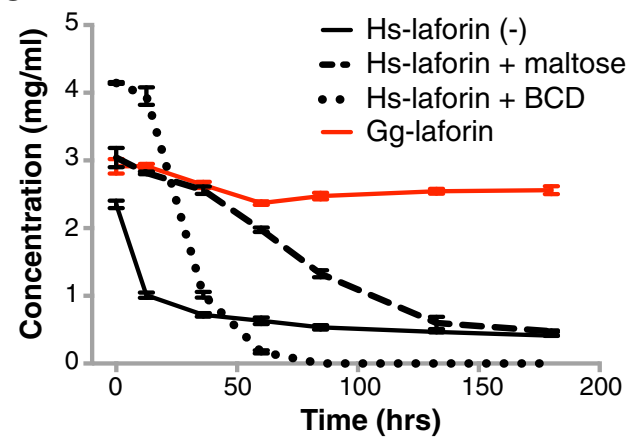

Figure 1 Purification and stability analysis of Hs-laforin and Gg-laforin. A. Hs-laforin was expressed in E. coli and purified by affinity chromatography in the absence (-) and presence of 15\% maltose or $10 \mathrm{mM} \beta$-cyclodextrin (BCD). Fractions of the pellet (P) and supernatant (S) after high-speed centrifugation and fractions of the IMAC elution (E) were analyzed by SDS-PAGE and stained with Coomassie Blue dye. B. Elution fractions from Hs-laforin and Gg-laforin preparations were concentrated using centrifugal filter units. Volume and concentration of each preparation were monitored throughout centrifugation, and protein concentration was measured using a Bradford assay. Total protein content for each preparation before and after concentration and the percent reduced were calculated for each preparation. C. Elution fractions were concentrated to approximately $2-4 \mathrm{mg} / \mathrm{ml}$ and incubated at room temperature for eight days. The concentration of each protein was measured during the course of the experiment using a Bradford assay. decrease as rapidly, confirming that the addition of maltose improves the stability of laforin over long periods of time. The addition of $\mathrm{BCD}$ improved the stability of laforin in the first 12 hours, but subsequently the concentration rapidly decreased and Hs-laforin in the presence of BCD became completely insoluble after 85 hours. Crystallography often demands that proteins be stable at high concentrations and for extended periods of time. These data demonstrate that the addition of BCD or maltose inhibits Hs-laforin from precipitating. While these results represent an improvement over previously reported Hslaforin purification strategies, crystallization trials in our lab have demonstrated that the presence of BCD or maltose inhibits Hs-laforin crystallization, possibly due to increased heterogeneity in the sample (unpublished data).

While the addition of maltose or BCD increases the stability of Hs-laforin, in addition to inhibiting crystallization, the presence of a sugar additive would interfere with glucan binding experiments and other biophysical assays. Therefore, we set out to identify a laforin ortholog that is similar to Hs-laforin, but more stable in vitro. Sequences of Hs-laforin and laforin orthologs from Mus musculus (mouse), Gallus gallus (red rooster), Хenopus tropicalis (frog), Anolis carolinensis (lizard) and Danio rerio (zebrafish) were aligned using ClustalW. Each of these orthologs contains the four invariant aromatic residues characteristic of a laforin CBM (Hs-laforin F5, W32, W60, and W99) and the signature DSP amino acid sequence, $\mathrm{DX}_{30} \mathrm{CX}_{2} \mathrm{GX}_{2} \mathrm{R}$ (Figure 2A). Additionally, these orthologs are $72-95 \%$ similar to Hs-laforin at the amino acid level (Figure 2B).

We obtained cDNA clones of the EPM2A gene from Mus musculus (Mm-laforin), Xenopus tropicalis (Xt-laforin), and Gallus gallus (Gg-laforin). Recombinant Mm-laforin was expressed with a $\mathrm{His}_{6}$-tag, and Xt-laforin was expressed as a $\mathrm{His}_{6}-\mathrm{SUMO}$ fusion protein in E. coli. Mm-laforin and $\mathrm{Xt}$-laforin were purified in the absence of any sugars and these preparations yielded more soluble protein than Hs-laforin, 6 and $10 \mathrm{mg} / \mathrm{L}$ of $E$. coli, respectively. However, the yield for Mm-laforin was not significantly greater than Hs-laforin, and Xt-laforin exhibited the same tendency as Hs-laforin to aggregate and precipitate (Additional file 1: Figure S1). Gg-laforin was also expressed as a $\mathrm{His}_{6}-\mathrm{SUMO}$ fusion protein and purified in the absence of any sugars. Gg-laforin purifications yielded approximately $14 \mathrm{mg} / \mathrm{L}$ of E. coli, a vast improvement compared to Hs-laforin. We then investigated the in vitro stability of recombinant Gg-laforin using the same assays as described for Hs-laforin. We found that Gg-laforin in the absence of any additive can be concentrated to over $18 \mathrm{mg} / \mathrm{ml}$, and the protein is stable $>180$ hours (Figure 1B-C). Thus, Gg-laforin is much less prone to precipitation compared to Hs-laforin at high concentrations and over long periods, and is more favorable for use in downstream biophysical methods. 


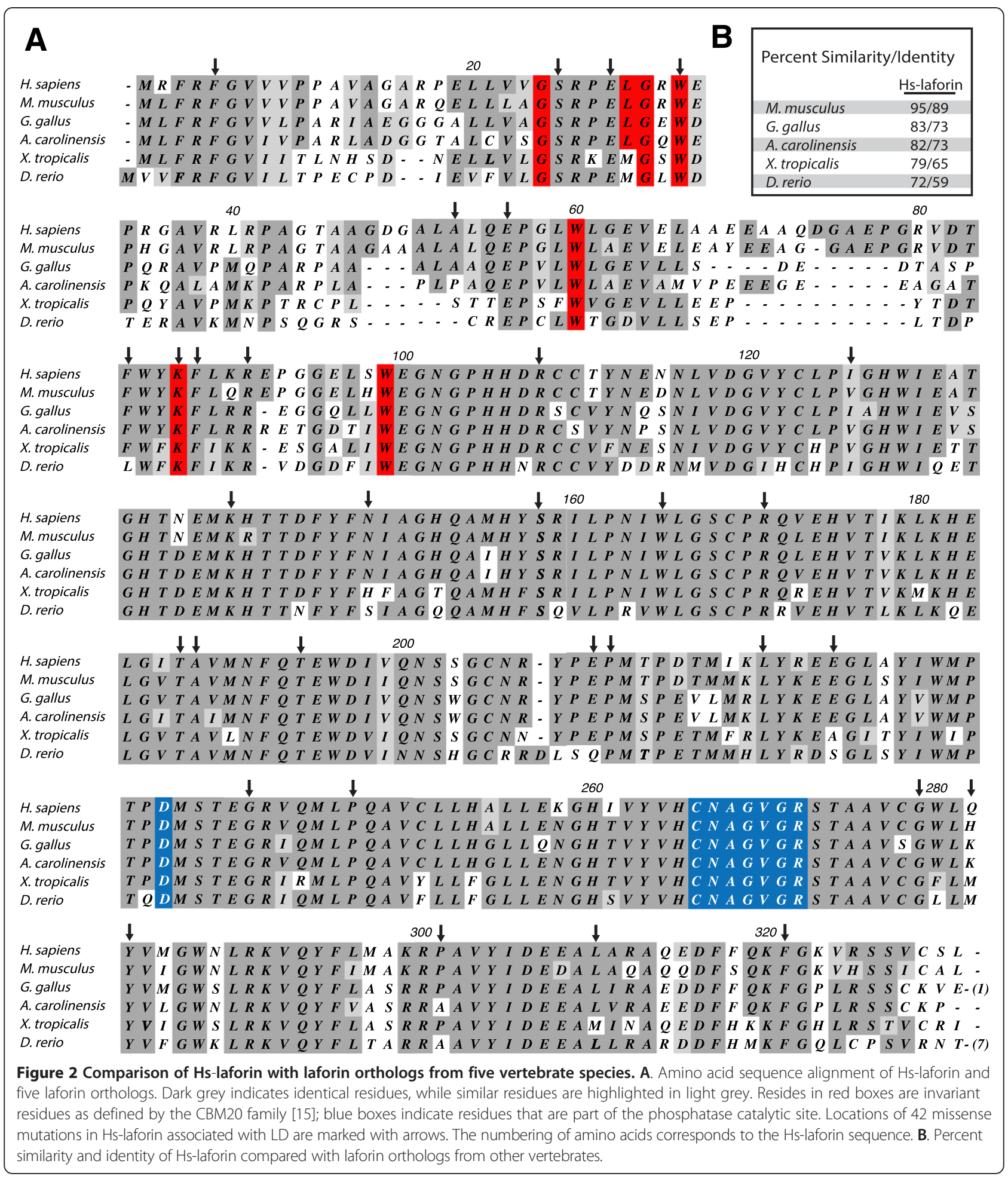

Gg-laforin purification yields a monomeric species

Given recent reports that full-length Hs-laforin cannot be purified as a soluble protein and our data demonstrating its instability, we sought to optimize Gg-laforin purification and to test its biochemical properties to determine whether Gg-laforin would be a good alternative for solving the laforin structure [27,28]. Recombinant $\mathrm{His}_{6}$-SUMO-Gg-laforin was expressed and purified from $E$. coli by affinity chromatography, digested with ULP1 to cleave the $\mathrm{His}_{6}-\mathrm{SUMO}$ tag, and subjected to reverse affinity chromatography to remove the tag and $\mathrm{His}_{6}$-tagged ULP1. These steps yielded 10 mg of untagged Gg-laforin per L of bacterial culture. 
Hs-laforin has a propensity to dimerize and form multimers $[8,30]$. In addition to a multimer peak, Hs-laforin elutes from size exclusion columns as a second peak with a small shoulder of larger molecular weight $[8,30]$. The small shoulder contains dimerized Hs-laforin and the major peak to the right of this shoulder is monomeric Hs-laforin [30]. In order to determine whether Gg-laforin also forms higher order species, Gg-laforin was subjected to size exclusion chromatography using a Superdex 200 column. Similar to Hs-laforin, Gg-laforin eluted as multiple peaks with a significant amount of protein in the multimer peak (Figure 3A). The chromatogram for the Gg-laforin elution showed a similar pattern as previously reported for Hs-laforin with both a dimer shoulder $(\sim 72 \mathrm{kDa})$ and a monomer peak $(\sim 36 \mathrm{kDa})$ (Figure 3A). Approximately $5 \mathrm{mg}$ of monomeric Gg-laforin was recovered from the size-exclusion elution fractions. To test if Gg-laforin exists in a dynamic monomer/dimer state, we collected the fractions from the monomer peak, concentrated the fractions, and re-loaded these fractions over the same column. Gg-laforin eluted as a $36 \mathrm{kDa}$ protein, and no dimer shoulder was present during this second purification, suggesting that monomeric Gg-laforin does not convert to a dimer (Figure 3B). The protein content and purity of the Superdex 200 monomeric fraction was assessed by collecting fractions and analyzing them by SDS-PAGE. Gg-laforin purified via this multi-step protocol migrated as a highly pure $36 \mathrm{kDa}$ protein (Figure $3 \mathrm{C}$ ). Previous studies have shown that Hslaforin dimers are resistant to SDS denaturation to a small extent, but there was no indication from the gel that a Gg-laforin dimer species was present [30,35]. To further define the size and oligomeric state of Gg-laforin, the Superdex 200-purified Gg-laforin protein was analyzed

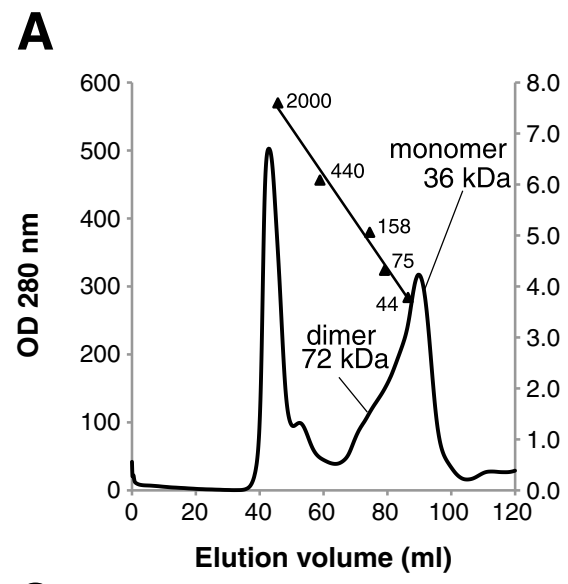

B
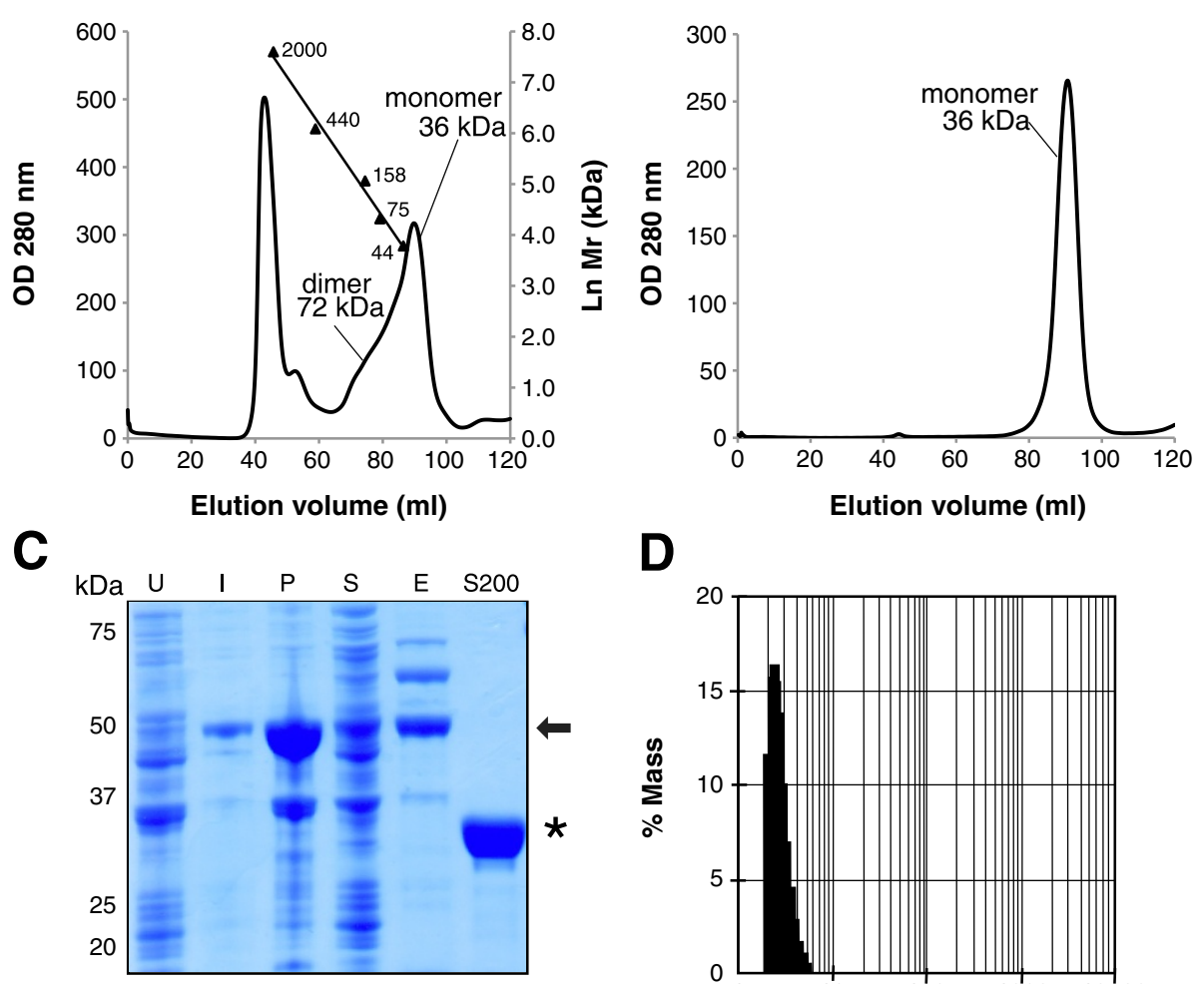

D

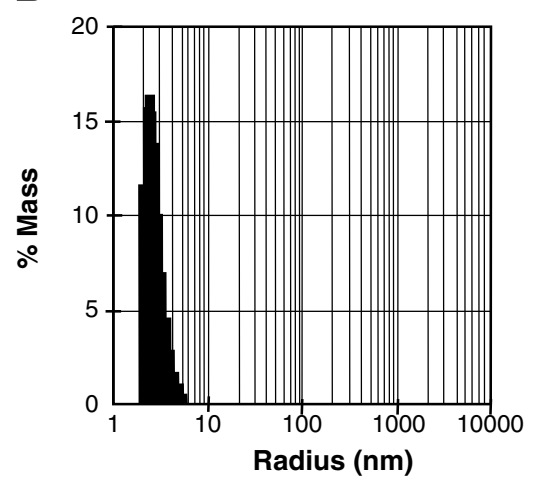

Figure 3 Purification and characterization of Gg-laforin. A. The chromatogram is of His 6 -SUMO- tagged Gg-laforin first purified using an IMAC affinity column, and then passed over a HiLoad 16/60 Superdex 200 size exclusion column. Calibration of molecular weight markers is indicated, and ordinates indicate the natural logarithm of molecular weight (Mr). B. The monomer fractions were collected, concentrated, and re-loaded over the same column. The chromatogram shows the results of this second round of size-exclusion chromatography. C. SDS-PAGE stained with Coomassie Blue of fractions from E. coli cells: (U) uninduced cells; (I) induced with IPTG; (P) pelleted/insoluble fraction; (S) soluble fraction; (E) IMAC eluate; and (S200) monomer fraction from Superdex 200 elution. $20 \mathrm{\mu g}$ of total protein was loaded per lane. His $\sigma_{6}-\mathrm{SUMO}-\mathrm{Gg}$-laforin runs as a $50 \mathrm{kDa}$ species until removal of the $\mathrm{His}_{6}-\mathrm{SUMO}$ tag after IMAC elution (indicated by the arrow). Untagged Gg-laforin is predicted to be $36 \mathrm{kDa}$ (indicated by the asterisk). D. Dynamic light scattering was performed on a $1 \mathrm{mg} / \mathrm{ml}$ sample of Superdex 200-purified Gg-laforin monomer using a Protein Solutions DynaPro-99 system. Scattering intensity was measured and presented as a fraction of the total protein mass. A single species was detected with a hydrodynamic radius of $2.68 \mathrm{~nm}$, corresponding to a molecular weight of $31.6 \pm 14.5 \mathrm{kDa}$. 
using dynamic light scattering (DLS). The hydrodynamic radius of the detected species corresponded to a $31.6 \pm$ $14.5 \mathrm{kDa}$ protein, the approximate size of the monomeric Gg-laforin (Figure 3D). Cumulatively, these data demonstrate that Gg-laforin can be cleaved from the $\mathrm{His}_{6}-\mathrm{SUMO}$ fusion tag, monomeric Gg-laforin can be resolved by sizeexclusion chromatography, and the monomers remain monomeric during subsequent chromatography steps. Thus, Gg-laforin behaves in a similar manner as previously reported for Hs-laforin $[7,8,30]$.

\section{Gg-laforin monomer binds glucans}

The CBM of Hs-laforin distinguishes this phosphatase from other protein tyrosine phosphatase superfamily members in that the CBM enables Hs-laforin to bind carbohydrates [37]. Gg-laforin is predicted to possess a CBM due to the high similarity between Hs-laforin and Gg-laforin in this region. The CBM of Gg-laforin is highly similar to the Hs-laforin CBM and was previously shown to bind glycogen in vitro [34]. Using agarose beads conjugated to the carbohydrate amylose, we investigated the glucan binding properties of Gg-laforin. The Vaccinia H1-related phosphatase (VHR) is a human phosphatase from the same DSP superfamily as laforin, but VHR lacks a CBM and is therefore unable to bind carbohydrates [8]. Hs-laforin, Gg-laforin and VHR were each incubated with amylose beads for $30 \mathrm{~min}$ at $4{ }^{\circ} \mathrm{C}$, the beads were then pelleted by centrifugation, the supernatant was removed, and the beads were treated with SDS-PAGE buffer to release the proteins bound to the beads. Subsequently, proteins in the supernatant were precipitated and resuspended in SDS-PAGE buffer. Proteins in the supernatant and pellet fractions were separated by SDS-PAGE and analyzed by Western blotting. Gg-laforin bound amylose to the same extent as Hs-laforin, and both were present almost entirely in the pellet (Figure 4A). Alternatively, VHR did not bind the amylose beads and remained in the supernatant as expected (Figure 4A). Thus, Gg-laforin possesses a CBM that is capable of binding amylose to a similar degree as Hs-laforin.

\section{Gg-laforin monomer has phosphatase activity comparable} to Hs-laforin

Another group reported that only Hs-laforin dimers possess phosphatase activity; however, work from our lab and others demonstrated that both monomer and dimer species of Hs-laforin are catalytically active [30,35]. To determine if monomeric Gg-laforin has similar activity as Hs-laforin, monomeric Gg-laforin was assayed for phosphatase activity using the artificial substrate para-nitrophenylphosphate (pNPP) over a range of $\mathrm{pH}$ values, from 5.0-8.0. Gg-laforin displayed similar specific activity to Hs-laforin and also, like Hs-laforin, displayed a preference for a lower $\mathrm{pH}$ (Figure 4B). Mutation of the catalytic cysteine residue
(C266) to serine within the DSP of Hs-laforin inactivates the enzyme $[8,38]$. We cloned and purified a corresponding Gg-laforin C253S mutant, and as expected this mutant displayed no activity and was used as a negative control.

Hs-laforin is the only human phosphatase known to bind and dephosphorylate glycogen and amylopectin

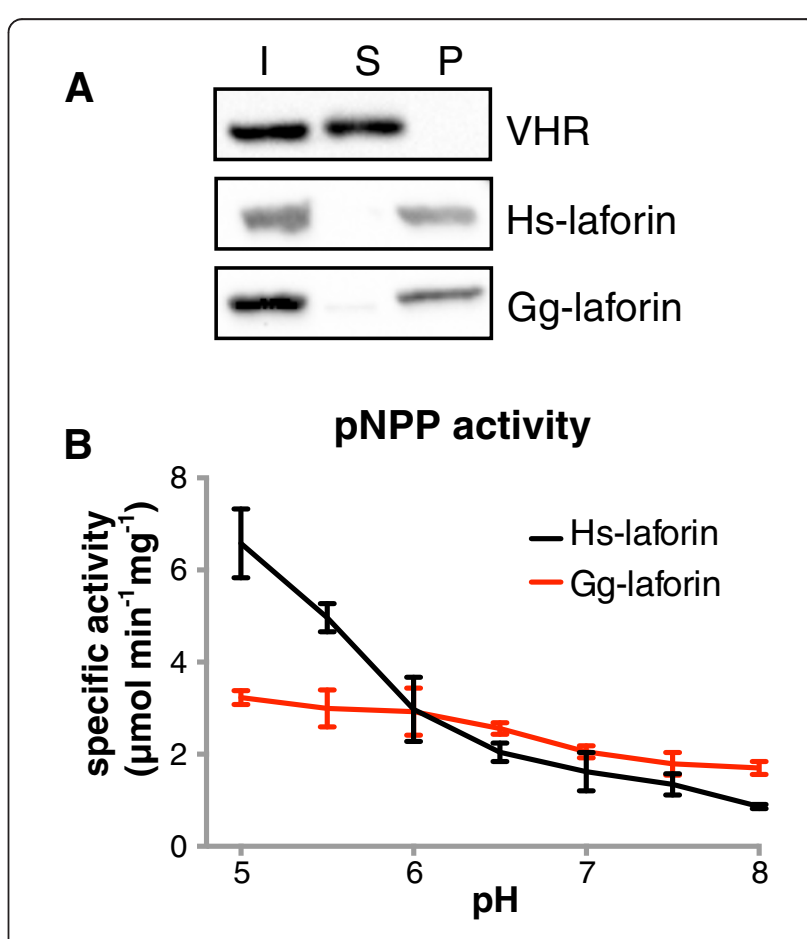

\section{C dephosphorylation of amylopectin}

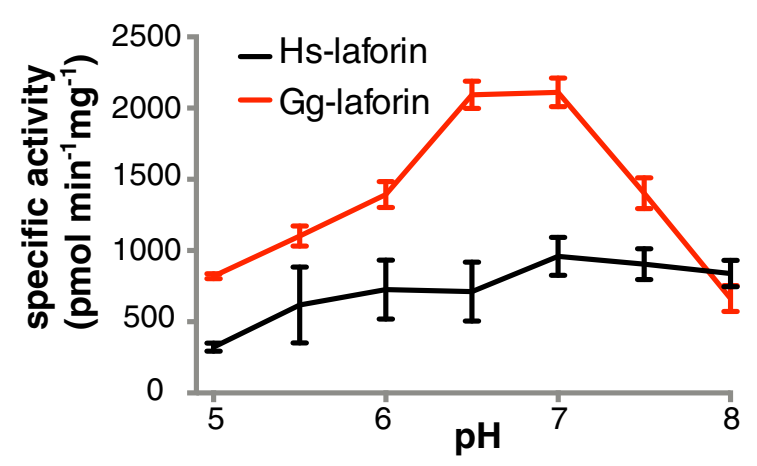

Figure 4 Analysis of carbohydrate binding and phosphatase activity of Gg-laforin. A. His-tagged proteins were incubated with amylose resin, then the amylose resin was pelleted by centrifugation, the supernatant was removed, and SDS-PAGE buffer was added to the pellet to release proteins bound to the amylose resin. Proteins in the supernatant were precipitated, and SDS-PAGE buffer was added to the supernatant sample. Protein input (I), supernatant (S) and pellet (P) samples were resolved by SDS-PAGE and visualized by Western analysis. B. Specific activities of Gg-laforin and Hs-laforin were quantified against the artificial substrate pNPP at pH units 5.0-8.0. C. Phosphate release from amylopectin using Gg-laforin and Hs-laforin at pH units 5.0-8.0 was measured using a malachite green assay. Error bars indicate mean \pm SD. 
in vitro $[8,16,17]$. Therefore, we investigated the ability of Gg-laforin to dephosphorylate the phosphorylated carbohydrate amylopectin using a malachite green-based assay that detects liberated inorganic phosphate [29]. Gg-laforin possesses higher specific activity against phosphorylated amylopectin than Hs-laforin, while preferring a similar $\mathrm{pH}$ to Hs-laforin (Figure 4C). These results demonstrate that Gg-laforin is a glucan phosphatase and an ortholog of Hs-laforin, interestingly with a somewhat greater ability to dephosphorylate glucans than Hslaforin. At the optimal $\mathrm{pH}, \mathrm{Gg}$-laforin has a lower specific activity against pNPP. Therefore, the two-fold increase in the specific activity of phosphate release from amylopectin may be due to differences in the CBM of Gg-laforin rather than differences between $\mathrm{Hs}$-laforin and Gg-laforin within the DSP. Indeed, the Hs-laforin and Gg-laforin DSP domains share $84 \%$ similarity, while the CBM of Gg-laforin is only $57 \%$ similar to the CBM of Hs-laforin [8]. However, most of the amino acids associated with LD mutations are conserved in the Gg-laforin CBM. These data show that Gg-laforin is a glucan phosphatase with similar activity levels as Hs-laforin, yet Gg-laforin is more soluble when purified as a fusion protein in a bacterial expression system.

\section{Conclusions}

Human laforin has proven to be a difficult protein to express in recombinant systems. These difficulties are highlighted by previous reports that Hs-laforin must be purified from inclusion bodies in E. coli or that only the Hs-laforin CBM is soluble in E. coli $[27,28]$. While structural information regarding the individual laforin domains would offer some insights into how laforin functions as a glucan phosphatase, the more intriguing questions focus on how the two domains are integrated and how they function synergistically during dephosphorylation of glycogen. Indeed, there are a number of structures of DSP domains and CBMs already determined, but due to the low degree of similarity with the laforin domains they do not offer much insight into the function of laforin [12,39-46]. We recently determined the structure of two Arabidopsis glucan phosphatases, Starch EXcess 4 (SEX4) and Like Sex Four 2 (LSF2). SEX4 contains a CBM and DSP domain, while LSF2 lacks a CBM. The individual laforin domains are likely to resemble the domains of SEX4 and LSF2 [32,33]. Indeed, laforin is functionally related to SEX4 and LSF2 (i.e. they are all glucan phosphatases); however, the DSP of laforin is $<39 \%$ similar to the DSP of SEX4 and LSF2, and the laforin CBM is from an entirely different sub-class of CBM than that of SEX4 [7,8,47-49]. Although SEX4 possesses a CBM and DSP, these domains are in the opposite orientation compared to laforin. SEX4 and LSF2 also each contain a C-terminal motif that integrally folds into the DSP and is essential for maintaining the integrity of the structure $[32,33]$. Although
SEX4 and LSF2 are the first glucan phosphatase structures to be determined, due to multiple differences in domain organization as well as degree of similarity these structures do not offer key insights into the structure of laforin.

Our lab has been successful in purifying sufficient amounts of Hs-laforin for in vitro assays without using denaturation and refolding steps, but recombinant Hslaforin has proved difficult to work with in experiments requiring large quantities of protein, due to low yields and the tendency to aggregate and precipitate. We sought a laforin ortholog with greater solubility and stability yet possessing similar in vitro characteristics as Hs-laforin; such an ortholog would be a more conducive target for crystallography and other biophysical techniques. The structure of this ortholog would provide insight into the mechanism of laforin function and may shed light on why mutations in certain amino acids lead to LD.

We have demonstrated that $\mathrm{His}_{6}$-SUMO-Gg-laforin is expressed as a soluble protein in E. coli, Gg-laforin remains soluble after cleavage of the fusion protein during experimental manipulation, and it possesses both phosphatase and glucan binding activity. Gg-laforin can be purified without the use of denaturation and refolding steps, and the protein does not require a sugar to improve its stability. We showed that Gg-laforin is present as a multimer and monomer, it remains monomeric after sizeexclusion chromatography, and it possesses phosphatase and glucan binding activity as a monomer. Monomeric Gg-laforin has robust phosphatase activity against the artificial substrate pNPP and also the more biologically relevant substrate amylopectin, similar to the activity of Hs-laforin as previously described $[7,8,30]$. Consequently, Gg-laforin is an excellent alternative to Hs-laforin for crystallization trials, and once determined, the structure of Gg-laforin will be a very good model for Hs-laforin in structure-function studies. The characterization of Gglaforin has provided an alternate route for obtaining the crystal structure of laforin that can be utilized to clarify the role of laforin in the metabolism of insoluble carbohydrates and the etiology of Lafora disease.

\section{Methods}

\section{Cloning procedures}

The ppSUMO plasmid was a generous gift from Dr. Jack Dixon (University of California, San Diego, USA). The plasmid pGL-EPM2A containing the gene for Mmlaforin (NP_034276.2) was a kind gift from Dr. Kazuhiro Yamakawa (Brain Science Institute, Wako-shi, Japan). Mm-laforin was subcloned into pET21a that includes a C-terminal $\mathrm{His}_{6}$ tag.

Expressed sequence tags (ESTs) of Xt-laforin (NP_001123695.1) and Gg-laforin (NP_001026240.1) were purchased from Open Biosystems and Delaware Biotechnology Institute, respectively, and cloned into ppSUMO 
according to standard protocols. ppSUMO encodes a small Ub-like modifier (SUMO) fusion tag that includes an amino-terminal $\mathrm{His}_{6}$-tag to aid purification. Sequences were verified by DNA sequencing. pET21a Vaccinia H1-related phosphatase (VHR) and pET21a Hs-laforin constructs have been described previously $[8,38]$.

\section{Protein expression and purification}

All proteins were expressed in BL21-CodonPlus E. coli cells (Stratagene) and purified using an IMAC column on a Profinia purification system (BioRad) followed by size exclusion chromatography. Bacterial cultures were grown in $1 \mathrm{~L} 2 \mathrm{xYT}$ or Terrific Broth (IBI Scientific) with $1 \mathrm{mM}$ kanamyacin and $1 \mathrm{mM}$ chloramphenicol at $37^{\circ} \mathrm{C}$ until $\mathrm{OD}_{600}$ reached $\sim 0.8$. Cultures were chilled on ice for 20 minutes, and isopropyl thio- $\beta$-D-galactopyranoside (IPTG) was added for a final concentration of $0.4 \mathrm{mM}$ to induce protein expression. After growth for approximately 12-16 hours, cells were harvested by centrifugation and stored at $-20^{\circ} \mathrm{C}$. Bacterial pellets expressing Hs-laforin were resuspended in buffer $\mathrm{A}$ : $50 \mathrm{mM}$ Tris/ $\mathrm{HCl}$ (pH 7.5), $300 \mathrm{mM} \mathrm{NaCl}$, and $2 \mathrm{mM}$ dithiothreitol (DTT). Pellets expressing Mm-laforin were resuspended in buffer B: $50 \mathrm{mM}$ Tris/ $\mathrm{HCl}$ (pH 8.0), $300 \mathrm{mM} \mathrm{NaCl}$, and $0.05 \% \beta$-mercaptoethanol. Pellets expressing VHR, Xtlaforin or Gg-laforin were resuspended in buffer C: $20 \mathrm{mM}$ Tris/ $\mathrm{HCl}$ (pH 7.5), $100 \mathrm{mM} \mathrm{NaCl}$ and $2 \mathrm{mM}$ DTT. $15 \%$ maltose $(\mathrm{w} / \mathrm{v})$ or $10 \mathrm{mM} \beta$-cyclodextrin was added to some preparations. Resuspended cells were lysed with a microfluidizer (EmulsiFlex-C5, Avestin), and soluble fractions were separated by high-speed centrifugation $(48,000 \mathrm{~g})$. $\mathrm{His}_{6}$-SUMO-tagged Xt-laforin and Gg-laforin were purified using a Profinia IMAC column (Bio-Rad) with a Profinia protein purification system (Bio-Rad) and dialyzed into buffer $\mathrm{C}$ in the presence of the SUMOspecific protease ULP1 that also contains a His 6 -tag. Reverse purification over the Profinia IMAC column was used to remove ULP1-His ${ }_{6}$ and the fusion tag. Each protein was then purified using a HiLoad 16/60 Superdex 200 size exclusion column and ÄKTA FPLC (GE Healthcare). Fractions containing the Gg-laforin monomer species were collected and put back over the same column. Mm-laforin, Hs-laforin and VHR were also expressed as $\mathrm{His}_{6}$-tagged recombinant proteins and purified in a similar manner.

\section{Protein gel electrophoresis, quantitation of stability, and dynamic light scattering}

Protein purity was assessed by sodium dodecyl sulfatepolyacrylamide gel electrophoresis (SDS-PAGE). Gels were stained with Coomassie brilliant blue to visualize proteins. To quantify stability of Hs-laforin and Gg-laforin, elution fractions were concentrated using centrifugal filter units (30 K, Amicon Ultra). Volume and concentration were monitored throughout centrifugation at 3,220 $\times g$, and protein concentration was measured using a Bradford assay. To test long-term stability, samples were concentrated to approximately $2-4 \mathrm{mg} / \mathrm{ml}$ and incubated at room temperature for eight days. Protein concentration was monitored during the course of the experiment using a Bradford assay.

Dynamic light scattering (Protein Solutions DynaPro99) was utilized to determine the hydrodynamic radius of particles in solution. The DLS system measures the size distribution of particles by detecting fluctuations in light intensity over time. Scattering intensity was presented as a fraction of the total protein mass; poly- or monodispersity in the sample was determined by the number of peaks on the DLS histogram. A standard curve embedded in the DLS software was used to calculate the approximate size of a globular protein with the observed hydrodynamic radius. Measurements were performed on a protein sample of $1 \mathrm{mg} / \mathrm{ml}$ at room temperature.

\section{Glucan binding assay}

Amylose immobilized on agarose resin (New England Biolabs) was pre-incubated with $1 \%$ BSA at room temperature for $30 \mathrm{~min}$ to prevent nonspecific binding. $0.25-1$ $\mu \mathrm{g}$ of each recombinant $\mathrm{His}_{6}$-tagged protein was mixed with $30 \mu \mathrm{l}$ amylose beads in buffer $C$ and protease inhibitor cocktail (2.5 mM AEBSF, $2.5 \mathrm{mM}$ benzamidine hydrochloride, $2.5 \mu \mathrm{M}$ leupeptin, $2.5 \mu \mathrm{M}$ E64) while rotating at $4^{\circ} \mathrm{C}$ for $30 \mathrm{~min}$. Amylose beads were pelleted by centrifugation $(2,300 \times g)$, the supernatant was removed, proteins in the supernatant were precipitated, and proteins in the pellet and supernatant were visualized by Western analysis. Blots were probed with mouse anti-His 6 1:4000 (NeuroMabs) and goat anti-mouse HRP (Invitrogen). SuperSignal West Pico (Thermo Scientific) was used to detect the HRP signal.

\section{Phosphatase assays}

Phosphatase activity was determined using the substrates para-nitrophenylphosphate (pNPP) and potato amylopectin as described previously $[8,29,30]$. The pNPP reactions were carried out in $50 \mu \mathrm{l}$ reactions in $1 \times$ phosphate buffer $(0.1 \mathrm{M}$ sodium acetate, $0.05 \mathrm{M}$ bisTris, $0.05 \mathrm{M}$ Tris- $\mathrm{HCl}$, and $2 \mathrm{mM}$ DTT at the appropriate $\mathrm{pH}), 50 \mathrm{mM}$ pNPP, and $200-400 \mu \mathrm{g}$ enzyme at $37^{\circ} \mathrm{C}$ for $2 \mathrm{~min}$. Reactions were terminated with the addition of $200 \mu \mathrm{l} 0.25 \mathrm{M} \mathrm{NaOH}$. Absorbance was measured at $410 \mathrm{~nm}$. Malachite green reactions were carried out in $20 \mu \mathrm{l}$ reactions in $1 \times$ phosphate buffer, $45 \mu \mathrm{g}$ amylopectin, and $100 \mathrm{ng}$ enzyme at $37^{\circ} \mathrm{C}$. After $2-5$ minutes, $20 \mu \mathrm{l}$ $0.1 \mathrm{MN}$-ethylmaleimide and $80 \mu \mathrm{l}$ malachite green reagent was added to quench the reaction, and absorbances were measured at $620 \mathrm{~nm}$ after 40 minutes. 
Assays were performed in triplicate for each enzyme at pH 5.0, 5.5, 6.0, 6.5, 7.0, 7.5, 8.0.

\section{Sequence alignment}

Amino acid sequences of laforin orthologs were obtained from NCBI, aligned by ClustalW [50], and refined manually using MacVector. LD missense mutations are marked according to listings in the Lafora Progressive Myoclonus Epilepsy Mutation and Polymorphism database (http:// projects.tcag.ca/lafora/) [51].

\section{Additional file}

Additional file 1: Figure S1. Multimerization of Xt-laforin. Xt-laforin was purified by IMAC and passed over a HiLoad 16/60 Superdex 200 size exclusion column. The chromatogram shows a prominent peak corresponding to a multimeric species and unresolved peaks corresponding to the Xt-laforin dimer and monomer (72 kDa and $36 \mathrm{kDa}$, respectively).

\section{Abbreviations}

LD: Lafora disease; LB: Lafora body; CBM: Carbohydrate-binding module; DSP: Dual-specificity phosphatase; SUMO: Small ubiquitin-like modifier; EST: Expressed sequence tag; VHR: Vaccinia H1-related phosphatase;

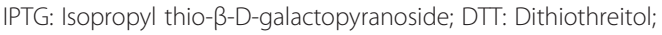
ULP1: Ubiquitin-like specific protease-1; pNPP: para-nitrophenylphosphate; DLS: Dynamic light scattering; BCD: $\beta$-cyclodextrin; IMAC: Immobilized metal affinity chromatography; SDS-PAGE: Sodium dodecyl sulfate-polyacrylamide gel electrophoresis.

\section{Competing interests}

The authors declare that they have no competing interests.

\section{Authors' contributions}

MSG conceived the study. MKB, WD, MBJ, and MSG carried out the cloning of laforin orthologs. WD expressed and purified Mm-laforin. MKB and SH carried out expression, purification and characterization of Hs-laforin, Xt-laforin and Gg-laforin. MKB and MSG wrote the manuscript. All authors read and approved the final manuscript.

\section{Acknowledgements}

We thank Dr. Carol Beach (University of Kentucky) for her assistance with the mass spectrometry as well as Dr. Martin Chow and the Center for Structural Biology (supported by NCRR grant P20RR0202171). This work was supported by NIH grant R01NS070899, an award from the Mizutani Foundation for Glycoscience, University of Kentucky College of Medicine startup funds to MSG, an American Heart Association Postdoctoral Award (12POST12030381) to WD, and a Summer Research Grant to MKB from the Office of Undergraduate Research at the University of Kentucky.

Received: 14 October 2013 Accepted: 27 March 2014

Published: 2 April 2014

\section{References}

1. Minassian BA: Lafora's disease: towards a clinical, pathologic, and molecular synthesis. Pediatr Neurol 2001, 25(1):21-29.

2. Gentry MS, Roma-Mateo C, Sanz P: Laforin, a protein with many faces: glucan phosphatase, adapter protein, and others. FEBS J 2013, 280(2):525:37.

3. Gentry MS, Dixon JE, Worby CA: Lafora disease: insights into neurodegeneration from plant metabolism. Trends Biochem Sci 2009, 34(12):628-639.

4. Delgado-Escueta AV: Advances in lafora progressive myoclonus epilepsy. Curr Neurol Neurosci Rep 2007, 7(5):428-433.

5. Ganesh S, Puri R, Singh S, Mittal S, Dubey D: Recent advances in the molecular basis of Lafora's progressive myoclonus epilepsy. J Hum Genet 2006, 51(1):1-8.
6. Singh S, Ganesh S: Lafora progressive myoclonus epilepsy: a meta-analysis of reported mutations in the first decade following the discovery of the EPM2A and NHLRC1 genes. Hum Mutat 2009, 30(5):715-723.

7. Gentry MS, Pace RM: Conservation of the glucan phosphatase laforin is linked to rates of molecular evolution and the glycogen metabolism of the organism. BMC Evol Biol 2009, 9(1):138.

8. Gentry MS, Dowen RH 3rd, Worby CA, Mattoo S, Ecker JR, Dixon JE: The phosphatase laforin crosses evolutionary boundaries and links carbohydrate metabolism to neuronal disease. J Cell Biol 2007, 178(3):477-488.

9. Wang J, Stuckey JA, Wishart MJ, Dixon JE: A unique carbohydrate binding domain targets the lafora disease phosphatase to glycogen. J Biol Chem 2002, 277(4):2377-2380.

10. Minassian BA, Lee JR, Herbrick JA, Huizenga J, Soder S, Mungall AJ, Dunham I, Gardner R, Fong CY, Carpenter S, Jardim L, Satishchandra P, Andermann E, Snead OC III, Lopes-Cendes I, Tsui LC, Delgado-Escueta AV, Rouleau GA, Scherer SW: Mutations in a gene encoding a novel protein tyrosine phosphatase cause progressive myoclonus epilepsy. Nat Genet 1998, 20(2):171-174.

11. Serratosa JM, Gomez-Garre P, Gallardo ME, Anta B, de Bernabe DB, Lindhout D, Augustijn PB, Tassinari CA, Malafosse RM, Topcu M, Grid D, Dravet C, Berkovic SF, de Córdoba SR: A novel protein tyrosine phosphatase gene is mutated in progressive myoclonus epilepsy of the Lafora type (EPM2). Hum Mol Genet 1999, 8(2):345-352.

12. Boraston $A B$, Bolam DN, Gilbert HJ, Daview GJ: Carbohydrate-binding modules: fine-tuning polysaccharide recognition. Biochem J 2004, 382:769-781.

13. Christiansen C, Hachem MA, Glaring MA, Vikso-Nielsen A, Sigurskjold BW, Svensson B, Blennow A: A CBM20 low-affinity starch-binding domain from glucan, water dikinase. FEBS Lett 2009, 583(7):1159-1163.

14. Machovic M, Janecek S: Starch-binding domains in the post-genome era. Cell Mol Life Sci 2006, 63(23):2710-2724.

15. Janecek S, Svensson B, MacGregor EA: Structural and evolutionary aspects of two families of non-catalytic domains present in starch and glycogen binding proteins from microbes, plants and animals. Enzym Microb Technol 2011, 49(5):429-440.

16. Worby CA, Gentry MS, Dixon JE: Laforin: A dual specificity phosphatase that dephosphorylates complex carbohydrates. J Biol Chem 2006, 281(41):30412-30418.

17. Tagliabracci VS, Turnbull J, Wang W, Girard JM, Zhao X, Skurat AV, Delgado-Escueta AV, Minassian BA, Depaoli-Roach AA, Roach PJ: Laforin is a glycogen phosphatase, deficiency of which leads to elevated phosphorylation of glycogen in vivo. Proc Natl Acad Sci U S A 2007, 104(49):19262-19266.

18. Tagliabracci VS, Girard JM, Segvich D, Meyer C, Turnbull J, Zhao X Minassian BA, Depaoli-Roach AA, Roach PJ: Abnormal metabolism of glycogen phosphate as a cause for lafora disease. J Biol Chem 2008, 283(49):33816-33825.

19. Tagliabracci VS, Heiss C, Karthik C, Contreras CJ, Glushka J, Ishihara M, Azadi P, Hurley TD, DePaoli-Roach AA, Roach PJ: Phosphate incorporation during glycogen synthesis and Lafora disease. Cell Metab 2011, 13(3):274-282.

20. Roach PJ, Skurat AV, Harris RA: Regulation of glycogen metabolism. In The Endocrine Pancreas and Regulation of Metabolism. Edited by Jefferson LS, Cherrington AD. New York, NY: Oxford University Press, Inc; 2001:609-647.

21. Lomako J, Lomako WM, Kirkman BR, Whelan WJ: The role of phosphate in muscle glycogen. Biofactors 1994, 4(3-4):167-171.

22. Lomako J, Lomako WM, Whelan WJ, Marchase RB: Glycogen contains phosphodiester groups that can be introduced by UDPglucose: glycogen glucose 1-phosphotransferase. FEBS Lett 1993, 329(3):263-267.

23. Nitschke F, Wang P, Schmieder P, Girard JM, Awrey DE, Wang T, Israelian J, Zhao X, Turnbull J, Heydenreich M, Kleinpeter E, Steup M, Minassian BA: Hyperphosphorylation of glucosyl C6 carbons and altered structure of glycogen in the neurodegenerative epilepsy lafora disease. Cell Metab 2013, 17(5):756-767.

24. Roach PJ: Are there errors in glycogen biosynthesis and is laforin a repair enzyme? FEBS Lett 2011, 585(20):3216-3218.

25. Roach PJ, Depaoli-Roach AA, Hurley TD, Tagliabracci VS: Glycogen and its metabolism: some new developments and old themes. Biochem J 2012, 441(3):763-787.

26. Tiberia E, Turnbull J, Wang T, Ruggieri A, Zhao XC, Pencea N, Israelian J, Wang Y, Ackerley CA, Wang P, Liu Y, Minassian BA: Increased laforin and laforin 
binding to glycogen underlie Lafora body formation in malin-deficient Lafora disease. J Biol Chem 2012, 287(30):25650-25659.

27. Castanheira P, Moreira S, Gama M, Faro C: Escherichia coli expression, refolding and characterization of human laforin. Protein Expr Purif 2010, 71(2):195-199

28. Moreira S, Castanheira P, Casal M, Faro C, Gama M: Expression of the functional carbohydrate-binding module (CBM) of human laforin. Protein Expr Purif 2010, 74(2):169-174.

29. Sherwood AR, Paasch BC, Worby CA, Gentry MS: A malachite green-based assay to assess glucan phosphatase activity. Anal Biochem 2013, 435(1):54-56.

30. Dukhande W, Rogers DM, Roma-Mateo C, Donderis J, Marina A, Taylor AO, Sanz $\mathrm{P}$, Gentry MS: Laforin, a dual specificity phosphatase involved in Lafora disease, is present mainly as monomeric form with full phosphatase activity. PLoS One 2011, 6(8):e24040.

31. Gentry MS, Worby CA, Dixon JE: Insights into Lafora disease: malin is an E3 ubiquitin ligase that ubiquitinates and promotes the degradation of laforin. Proc Natl Acad Sci U S A 2005, 102(24):8501-8506.

32. Vander Kooi CW, Taylor AO, Pace RM, Meekins DA, Guo HF, Kim Y, Gentry MS: From the Cover: Structural basis for the glucan phosphatase activity of Starch Excess4. Proc Natl Acad Sci U S A 2010, 107(35):15379-15384.

33. Meekins DA, Guo HF, Husodo S, Paasch BC, Bridges TM, Santelia D, Kotting O, Vander Kooi CW, Gentry MS: Structure of the Arabidopsis glucan phosphatase like sex four2 reveals a unique mechanism for starch dephosphorylation. Plant Cell 2013, 25(6):2302-2314.

34. Ganesh S, Tsurutani N, Suzuki T, Hoshii Y, Ishihara T, Delgado-Escueta AV, Yamakawa K: The carbohydrate-binding domain of Lafora disease protein targets Lafora polyglucosan bodies. Biochem Biophys Res Commun 2004, 313(4):1101-1109.

35. Liu Y, Wang Y, Wu C, Liu Y, Zheng P: Dimerization of Laforin is required for its optimal phosphatase activity, regulation of GSK3beta phosphorylation, and Wnt signaling. J Biol Chem 2006, 281(46):34768-34774.

36. Roma-Mateo C, Solaz-Fuster MC, Gimeno-Alcaniz JV, Dukhande W, Donderis J, Marina A, Criado O, Koller A, Rodriguez De Cordoba S, Gentry MS, Sanz P: Laforin, a dual specificity protein phosphatase involved in Lafora disease, is phosphorylated at Ser25 by AMP-activated protein kinase. Biochem J 2011, 439(2):265-275.

37. Tonks NK: Protein tyrosine phosphatases: from genes, to function, to disease. Nat Rev Mol Cell Biol 2006, 7(11):833-846.

38. Denu JM, Zhou G, Wu L, Zhao R, Yuvaniyama J, Saper MA, Dixon JE: The Purification and Characterization of a Human Dual-specific Protein Tyrosine Phosphatase. J Biol Chem 1995, 270(8):3796-3803.

39. Xiao J, Engel JL, Zhang J, Chen MJ, Manning G, Dixon JE: Structural and functional analysis of PTPMT1, a phosphatase required for cardiolipin synthesis. Proc Natl Acad Sci U S A 2011, 108(29):11860-11865.

40. Lee J-O, Yang H, Georgescu M-M, Di Cristofano A, Maehama T, Shi Y, Dixon JE, Pandolfi P, Pavletich NP: Crystal Structure of the PTEN Tumor Suppressor: Implications for Its Phosphoinositide Phosphatase Activity and Membrane Association. Cell 1999, 99(3):323-334.

41. Yuvaniyama J, Denu JM, Dixon JE, Saper MA: Crystal structure of the dual specificity protein phosphatase VHR. Science 1996, 272(5266):1328-1331.

42. Tung JY, Chang MD, Chou WI, Liu YY, Yeh YH, Chang FY, Lin SC, Qiu ZL, Sun YJ: Crystal structures of the starch-binding domain from Rhizopus oryzae glucoamylase reveal a polysaccharide-binding path. Biochem J 2008, 416(1):27-36.

43. Boraston AB, Healey M, Klassen J, Ficko-Blean E, Lammerts Van Bueren A, Law V: A structural and functional analysis of alpha-glucan recognition by family 25 and 26 carbohydrate-binding modules reveals a conserved mode of starch recognition. J Biol Chem 2006, 281(1):587-598.

44. Sorimachi K, Le Gal-Coeffet MF, Williamson G, Archer DB, Williamson MP. Solution structure of the granular starch binding domain of Aspergillus niger glucoamylase bound to beta-cyclodextrin. Structure 1997, 5(5):647-661.

45. Steichen JM, Petty RV, Sharkey TD: Domain characterization of a 4-alphaglucanotransferase essential for maltose metabolism in photosynthetic leaves. J Biol Chem 2008, 283(30):20797-20804.

46. Koveal D, Clarkson MW, Wood TK, Page R, Peti W: Ligand binding reduces conformational flexibility in the active site of tyrosine phosphatase related to biofilm formation A (TpbA) from Pseudomonasaeruginosa. J Mol Biol 2013, 425(12):2219-2231.

47. Santelia D, Kotting O, Seung D, Schubert M, Thalmann M, Bischof S, Meekins DA, Lutz A, Patron N, Gentry MS, Allain FH, Zeeman SC: The phosphoglucan phosphatase like sex Four2 dephosphorylates starch at the C3-position in Arabidopsis. Plant Cell 2011, 23(11):4096-4111.
48. Kotting O, Santelia D, Edner C, Eicke S, Marthaler T, Gentry MS, ComparotMoss S, Chen J, Smith AM, Steup M, Ritte G, Zeeman SC: STARCH-EXCESS4 Is a Laforin-Like Phosphoglucan Phosphatase Required for Starch Degradation in Arabidopsis thaliana. Plant Cell 2009, 21(1):334-346.

49. Hsu S, Kim Y, Li S, Durrant ES, Pace RM, Woods VL Jr, Gentry MS: Structural insights into glucan phosphatase dynamics using amide hydrogendeuterium exchange mass spectrometry. Biochemistry 2009, 48(41):9891-9902.

50. Thompson JD, Higgins DG, Gibson TJ: CLUSTAL W: improving the sensitivity of progressive multiple sequence alignment through sequence weighting, position-specific gap penalties and weight matrix choice. Nucl Acids Res 1994, 22(22):4673-4680.

51. lanzano L, Zhang J, Chan EM, Zhao X, Lohi H, Scherer SW, Minassian BA: Lafora progressive myoclonus epilepsy mutation database-EPM2A and NHLRC1 (EMP2B) genes. Hum Mutat 2005, 26(4):397.

doi:10.1186/1471-2091-15-8

Cite this article as: Brewer et al:: Expression, purification and

characterization of soluble red rooster laforin as a fusion protein in Escherichia coli. BMC Biochemistry 2014 15:8.

\section{Submit your next manuscript to BioMed Central and take full advantage of:}

- Convenient online submission

- Thorough peer review

- No space constraints or color figure charges

- Immediate publication on acceptance

- Inclusion in PubMed, CAS, Scopus and Google Scholar

- Research which is freely available for redistribution
( BioMed Central 